Monatsschrift f. Geburtshülfe u. Gynäkologie 1937;105:I-IV

\title{
Contents, Vol. 105, 1937
}

Inhaltsverzeichnis.

Originalarbeite $\pi$. Seite

Alovski, A., und Burceva, E., Die Transfusion von konserviertem

Blutplasma 38

Bak, Michael, „Kalt-Test”-Untersuchungen an Sehwangeren, Ge-

bärenden und Wöchnerinnen 24

Bickenbach, Nachruf Franqué

Bonilla, Francisco und Kramann, Heinr., Über thyreotropes Hormon

in der Schwangerschaft 8

Brander, T., Über intrakranielle Geburtsverletzungen im Anschluß an

Geburt in Beckenendlage 205

Brockmann, siehe Guthmann. Burceva, E., siehe Alvorski, A.

Dorr, Hans, Über einen Fall von Morbus Basedow und Schwanger

schaft 278

Endres, Phüipp, siehe Guthmann, Heinr.

Enneper, Friedrich, Die Ursachen der Frühgeburt unter besonderer

Berücksichtig·ung des konstitutionellen Gesichtspunktes. . . . 347 Erbslöh, Joachim, Über den vergeblichen Zangenversuch inner- und

außerhalb der Klinik 257

Feder, W., siehe Presser, H.

Frigyesi, Josef, Über die operative Behandlung der tuberkulösen

Adnexerkrankungen 193

Gold, Leo, Über funktionelle Uterusblutungen

Graf, Adolf, Die Fertilität der Frau nach Amputation und Paquelini-

sierung der Portio als Folge der Behandlung von Erosionen und

der Elongatio colli 272

Guthmann, Heinr., und Brockmann, Beckenneigungswinkel, Eintritts-

winkel und Beckenîorm bcim hohen Gerad- und Schrägstand 97 Guthmann, Heinr., und Endres, Phüipp, Beeinflußt der vorzeitige und

frühzeitige Blasensprung · die Geburtsdauer?

$21 \mathrm{H}$

Heimann, Fritz, Postoperative reflektorisehe Funktionsstörung der

Meren 283

Knebel, Rud., Systematisches instrumentelles Beckenmessen ... 65

Köhler, P., Behandlung von Frauenleiden in Moorbädern 333

Kramann, Heinr., siehe Bonilla, Francisco.

Mönch, G. L., Zu den neueren Gesichtspunkten der menschlichen

Fruchtbarkeit 154

Nürnberger, L., Schwangerschaftsglykosurie und Hormonstoffwechsel 1 Pallos, Karl, Beiträge zur Klinik und Pathologie der Granulosazellen- 
geschwülste 139

Pasztor, Mihaly, Über das ovarielle Asthma bronchiale 161

Plaas, Erwin, Eigenartige Verlaufsform einer Tubargravidität . . 359

IY Inhaltsverzeichnis.

S $\theta$ ito

Presser, H., und Feder, W., Erîahrungen mit der Flavadin-Behandlung

bei der Gonorrhoe der Trau 107

Rivoir, Johann, Das Vaginalsekret als Ätiologie der primären und

rezidivierenden Cystitis trigoni 344

de Snoo, K., Das trinkende Kind im Uterus 88

Ssarygin, J. J., Die Hydrothermomamminisation bei Gebärmutter-

blutungen $\quad 30$

Tasch, Hans, Ein Fall von Gürtelplazenta 326

Vajna, Gábor, Uterusruptur bei Ballonbehandlung • 17

Wínkler, Hellmuíh, Die Aschenstruktur der Uterusschleirahaut und

ihre mikrochemische Diîierenzierung $\quad 117$

Wiríh, L., Eklampsieschädigungen und Liquordruck ....... 225

Grenzgebiete der Geburtshílfe und Gynäkologie.

Cholezystitis, Cholelithiasis und Appendizitis. Von Dr. Hans Dwor-

zak-Prag $\quad 47$

Stoîf $>$ vechsel bei Schwangeren. Von Dr. O. Mühlbock-Amsterdam 53

Die Köntgentherapie in der Gynäkologie. Von August 1935 bis De-

zember 1936. Von Dr. med. Hans Bosch und Dr. Willi

Leupold-Erlangen 171

Physikalisehe Heilmethoden einschließlich der Hydrotherapie. Von

Hermann Franken-Freiburg 228

Die Erkrankungen der Leber, Niere und der ableitenden Harnwege in

ihren Beziehungen zur Geburtshilfe und Gynäkologie. Von Prof.

Dr. H. Rupp-Bonn 287

Kreislauîbericht über das Jahr 1936. Von Willi Wolf-Tubingen. . 363

Sitzungsberichte aus geburtshïlf Iich - gynäkologischen Gesellschaîten.

Tagung der Abteilung · iür Geburtshilfe und Gynäkologie der Gesell-schaît Deutseher Naturîorscher und Ärzte in Gemeinschaît mit der Mitteldeutschen und Südostdeutschen Gesellschaît für Geburtshilfe und Gynäkologie. Sitzung vom 19. September 1936. 233

Gesellschaît für Geburtshilfe und Gynäkologie zu Berlin. Sitzung

vom 13. November $1936 \quad 188$

Gynäkologische Gesellschaît zu Breslau. Sitzung vom 13. Dezember

193561

Nordwestdeutsche Gesellschaît iür Gynäkologie. Sitzung vom 31. Ok-

tober 1936 in Hamburg 245

Mittelrheinische Gesellschaît îür Geburtshilîe und Gynäkologie.

Sitzung vom 6. Dezember $1936 \quad 300$

Geburtshilîlich-gynäkologische Gesellschaît in Wien. Sitzung vom

15. Dezember 1936190

-, Sitzung vom 12. Januar $1937 \quad 318$

-, Sitzung vom 9. Februar $1937 \quad 375$ 
-, Sitzung vom 9. März 1937377

Buchbespreehung. 64 